\title{
Amplitude Enhancement of Flow-Induced Vibration for Energy Harnessing
}

\author{
Ze Shao ${ }^{l,}{ }^{*}$, Tongming Zhou ${ }^{1}$, Hongjun $\mathrm{Zhu}^{2}$, Zhipeng Zang ${ }^{3}$ and Wenhua Zhao ${ }^{1}$ \\ ${ }^{1}$ Department of Civil, Environmental and Mining Engineering, The University of Western Australia, Perth, Australia \\ ${ }^{2}$ State Key Laboratory of Oil and Gas Reservoir Geology and Exploitation, Southwest Petroleum University, 8 Xindu Road, Chengdu, Sichuan \\ 610500, China \\ ${ }^{3}$ State Key Laboratory of Hydraulic Engineering Simulation and Safety, Tianjin University, Tianjin 300072, China
}

\begin{abstract}
In this paper, flow-induced vibrations of bluff bodies with four different cross-sectional geometries (circle, square, triangle and semi-circle) arranged both in single and tandem (gap ratio equals to 3 and 5) configurations are investigated in a wind tunnel. It is found that triangular and square cylinders have the higher amplitude than that of the semi-circular and the circular cylinders in the single configuration. When two cylinders are arranged in tandem, the circular cylinders have the highest amplitude among all tested cylinders. Furthermore, the semi-circular cylinder shows that its vibrating amplitude increases with the reduced velocity in the tandem system due to the galloping effect.
\end{abstract}

\section{Introduction}

The rapid growth of energy demand and the increasing concern of environmental degradation have attracted significant attention on renewable energy in recent years. Therefore, significant attention has been paid to renewable and clean energy resources. Compared to other renewable energy sources, the ocean seems to be a promising alternative as it covers more than $70 \%$ of the Earth's surface. Hence, various approaches for extracting fluid energy are being actively explored. One of these is to utilize the phenomenon of flowinduced vibrations (FIV), which include vortexinduced vibrations (VIV), galloping and wake-induced vibration (WIV), of a bluff structure to extract electrical energy from hydro-kinetic energy. Due to the periodic vortex shedding and the time-dependent pressure variation on the elastically mounted cylinder, the force exerted on the cylinder changes with time at a certain frequency. When the frequency of the vortex shedding $f_{v}$ is close to the natural frequency of the cylinder, an elastically mounted cylinder will start to vibrate due to resonance. This phenomenon is termed as VIV and the velocity region over which the vortex shedding frequency couples with the natural frequency of the cylinder is termed as the lock-on region. Another main phenomenon associated with FIV is galloping, which occurs on the cylinder with an asymmetry section. Unlike VIV, galloping is a velocity-dependent hydro-elastic instability characterized by lower frequencies and larger amplitudes than VIV [1]. This vibration response is significantly increased with the growth of the flow speed. It is more vigorous and destructive than VIV. Furthermore, galloping of the cylinder in FIV may also occur when it is located in the wake of an upstream cylinder, which is known as WIV [2].

Generally, FIV may result in catastrophic and instant damage to a structure. Thereby considerable research efforts have been focused on suppressing FIV. On the contrary, the large vibration amplitude of the cylinder means that high energy is contained in the vibrating body. Approaches on how to harness this kind of energy have attracted significant attention in recent years and substantial devices have been designed so far. One such example is the VortexInduced Vibration for Aquatic Clean Energy (VIVACE) converter with a circular cylinder subjected to a cross flow [3]. It was found that the vibration amplitude was an important parameter for the efficiency of the device, and indicated that the energy conversion efficiency was quite low because the vibration was only limited to the lock-on region. Therefore, methods to improve energy harnessing efficiency from galloping and WIV have attracted significant attentions in recent years. Sun et al. [4] investigated the VIVACE converter and concluded that cylinders in tandem configuration could harness power up to 13.49 times the power of a single cylinder system, and the efficiency of two cylinders was from 2 to 6.68 times that of a single cylinder. In fact, compared with the comprehensive reviews regarding the enhancement of FIV amplitude of circular cylinders, the investigation on other geometries with various arrangements regarding FIV energy harnessing are

\footnotetext{
*Corresponding author: ze.shao@research.uwa.edu.au
} 
relatively less. Therefore, this paper is aiming to investigate the influences on vibration amplitude of both single cylinder and tandem cylinders with different geometries (circular, semi-circular, triangle and square) for two streamwise gap ratios (centroid-tocentroid distance $G / D$ is 3 and 5). The geometry of the upstream and the downstream cylinders is kept the same in each test.

\section{Experiment set-up}

The tests were conducted in the wind tunnel at The University of Western Australia. A steel frame was set perpendicular to the wind direction (Figure 1). The tested cylinder with diameter (D) of $80 \mathrm{~mm}$ and length of $1600 \mathrm{~mm}$ was set orthogonally to the wind direction and installed upon the frame with four springs of equal stiffness of about $3100 \mathrm{~N} / \mathrm{m}$. Free decay tests were conducted first to obtained the natural frequency $f_{n}$ and the damping ratio $\zeta$. The main variables of the system are shown in Table 1. Furthermore, when conducting tests for two cylinders arranged in tandem configuration, another cylinder of the same geometry was fixed in front of the tested cylinder with $G / D=3$ and 5.

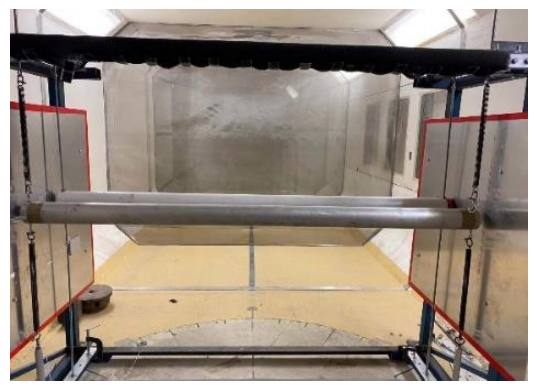

Figure 1. Experiment set-up

Table 1. Main parameters of the system

\begin{tabular}{ccccc}
\hline Parameters & Circular Cylinder & Triangular Cylinder & Semi-circular Cylinder & Square Cylinder \\
\hline Weight $(\mathrm{kg})$ & 2.1 & 5.4 & 3 & 7.6 \\
Mass ratio & 212.3 & 994.2 & 606.5 & 603.4 \\
Natural frequency $f_{n}$ & 6.6 & 5.3 & 5.6 & 4.5 \\
Damping ratio $\zeta$ & 0.0006 & 0.0004 & 0.0005 & 0.0003 \\
\hline
\end{tabular}

The cross-section of each geometry is shown in Figure 2. Also, except for the circular and square cylinders, the orientation of the triangular and semi-circular cylinders should be considered because the cross-sections of them are not symmetric with regards to the vertical plane. Figure 3 and Figure 4 explicate the arrangements of two tandem cylinders with different orientations.

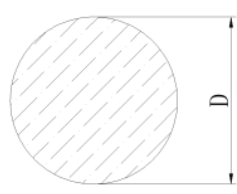

(a) Circular

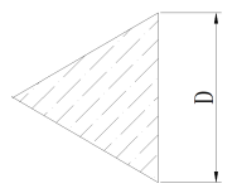

(b) Equilateral Triangular

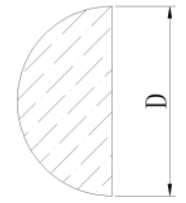

(c) Semi-circular

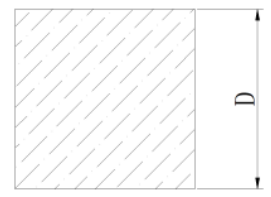

(d) Square

Figure 2. Cross-section of different bluff bodies

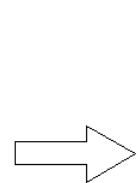

Flow

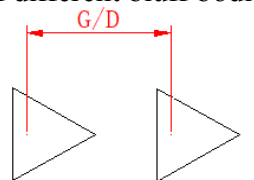

(a) Front to Back
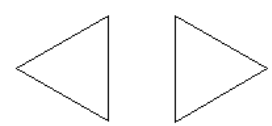

(b) Back to Back
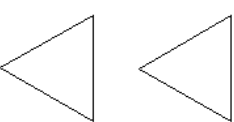

(c) Back to Front

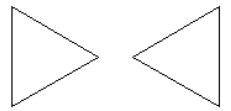

(d) Front to Front

Figure 3. Orientations of the triangular cylinders

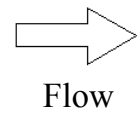

Flow

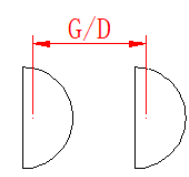

(a) Front to Back

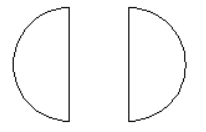

(b) Back to Back

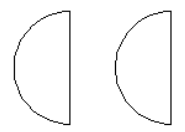

(c) Back to Front

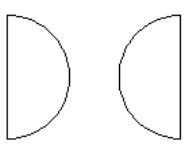

(d) Front to Front

Figure 4. Orientations of the semi-circular cylinders 


\section{Results and discussion}

The VIV responses of a single cylinder with different cross-sectional geometries were examined first and the results are shown in Figure 5. It can be seen that VIV occurs on the circular and semi-circular cylinders, showing an inertial branch, upper branch and lower branch. This result is consistent with that shown before $[5,6]$. For the circular cylinder, the maximum amplitude is about 0.53 , occurring at $U_{r}=7.1$, where $U_{r}$ is the reduced velocity, which is defined as $U_{r}=$ $U / D f_{n}$. This value is much smaller than that obtained in water due to the high mass ratio in air. The maximum value of $A / D$ for the semi-circular cylinder is about $7 \%$ larger than that for the circular cylinder, indicating that the semi-circular cylinder may be a better option than circular cylinder in terms of hydrodynamic energy harnessing based on VIV. However, as both of them show a lock-on region with comparable width, this characteristic indicates that VIV energy harnessing can only be conducted over a limited range of fluid velocity. For triangular and square cylinders, their vibration amplitudes increase with the increase of wind speed, coinciding with the characteristics of galloping. Both of them show a plateau with the triangular cylinder occurring earlier than the square one. The lock-on region of them are much wider than that of the circular and semi-circular cylinders, making them the good options for hydrodynamic energy harnessing based on VIV.

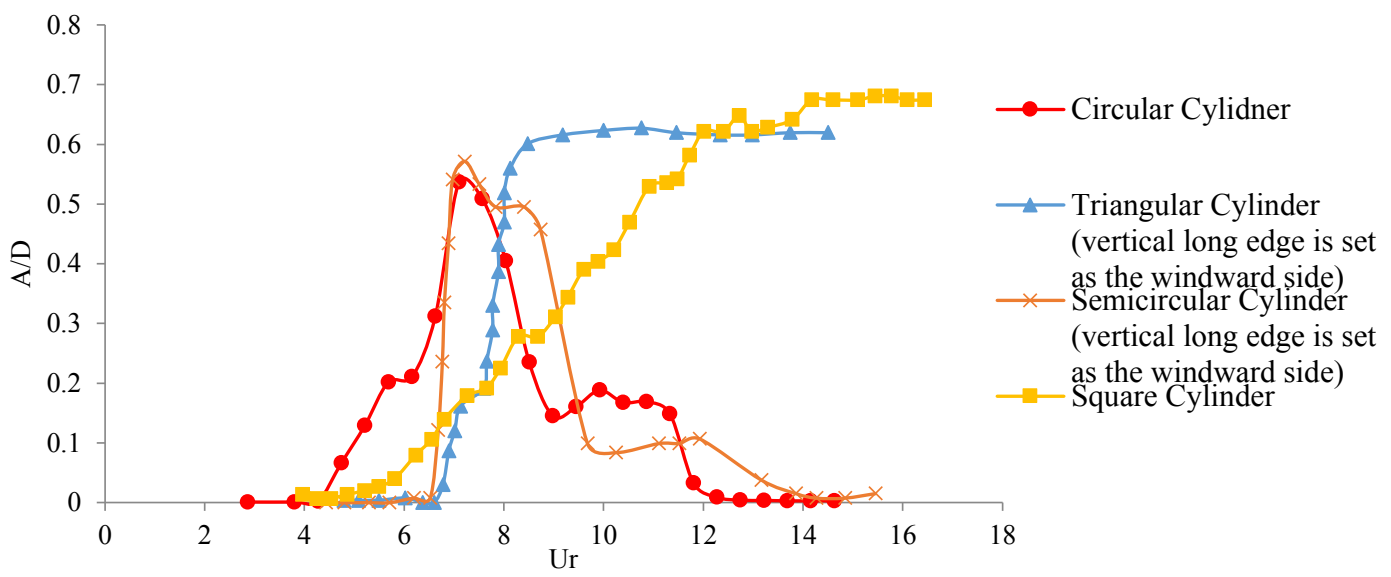

Figure 5. Comparison of vibration amplitude for different cylinders

When two cylinders are arranged in tandem, the cylinder will experience WIV (Figure 6). This may lead to two possibilities. The first one is that the VIV is still occurring in the first half of the synchronization region, however, galloping takes over after the end of the synchronization region (i.e. circular cylinders at gap ratio of 3 ). The second possibility is that VIV never happens in this case and only the early stage triggers unsteady galloping phenomenon with significantly $U_{r}$ (i.e. circular cylinders at gap ratio of 5). Previous studies showed that the reason for this phenomenon was related to the flow and vortex shedding characteristics of two tandem cylinders [7]. In the present study, two gap ratios are chosen as 3 and 5

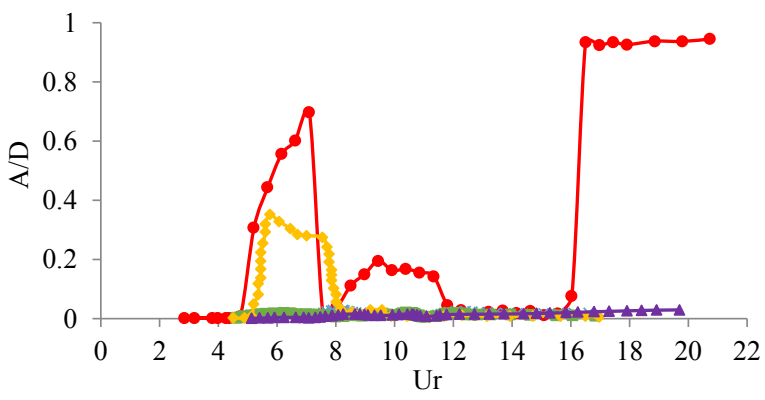

(a) Triangular cylinders at $G / D=3$ respectively, which corresponds to reattachment and co-shedding flow regimes. It is meant that the shear layers from the upstream cylinder may reattach on the downstream body and a vortex wake may not develop in the gap at low $U_{r}$ with gap ratio of 3 . Hence, the downstream cylinder starts vibration because of its own vortex shedding which seems like VIV. While increasing the reduced velocity or expanding the gap ratio with enough space between two cylinders for the vortex weak develops in the gap, the vibration becomes galloping as the downstream cylinder is sustained by the unsteady forces fluctuations caused by vortices shed from upstream body interacting with the shedding from the downstream one.

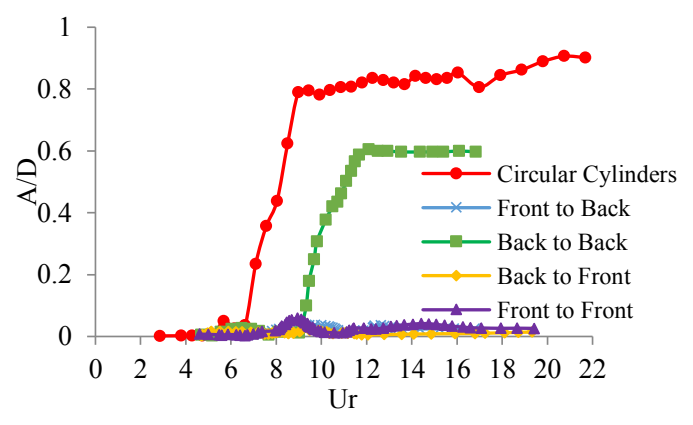

(b) Triangular cylinders at $G / D=5$ 


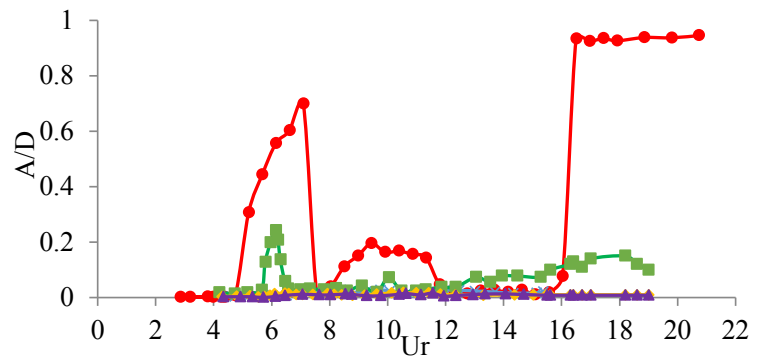

(c) Semi-circular cylinders at $G / D=3$

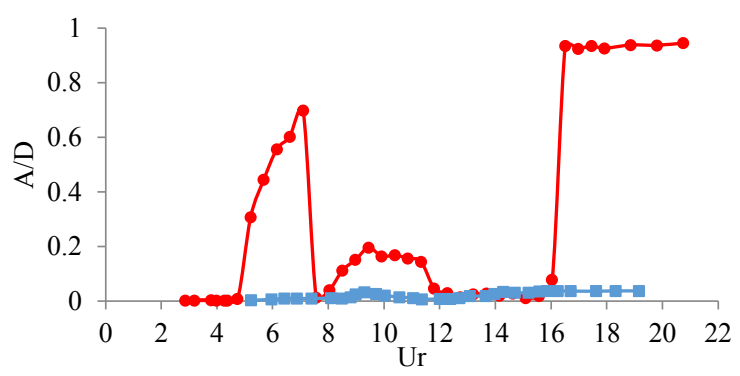

(e) Square cylinders at $G / D=3$

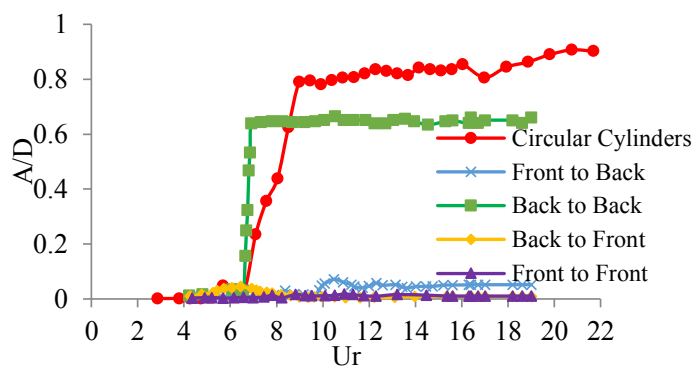

(d) Semi-circular cylinders at $G / D=5$

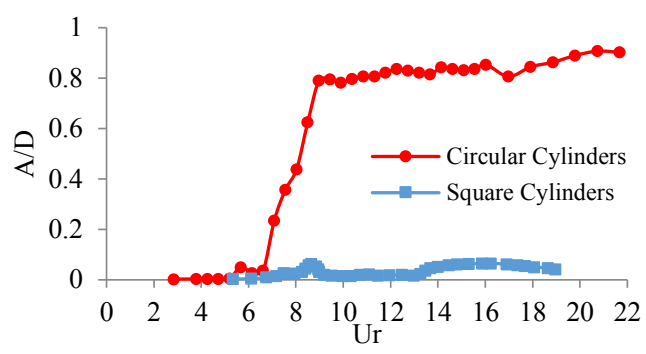

(f) Square cylinders at $G / D=5$

Figure 6. Comparsion of vibration amplitude for different cylinders arranged in tandem at gap ratios of 3 and 5

From Figures 6 (a) and (b), when two tandem triangular cylinders are arranged in "Back to Front" orientation (Figure $3(\mathrm{c})$ ) at gap ratio of 3 , the downstream cylinder starts vibrating at $U_{r}=4.8$, and the largest amplitude ratio is 0.36 . The lock-on region is limited to $U_{r}=5-8$. This could be because at this arrangement, the front cylinder has strong vortex shedding, which impinges on the front surfaces of the downstream cylinder, resulting in increased lift forces on it. In contrast, when triangular cylinders are set as Figure 3 (a) (b) and (d), the downstream one does not vibrate. Besides, tandem triangular cylinders which are set "Back to Back" orientation (Figure 3(d)) at gap ratio of 5 starts vibrating at $U_{r}=9$. After a quick increase with $U_{r}$, the amplitude keeps a constant of around 0.6. Furthermore, based on Figures 6 (c) and (d), WIV only happens on the downstream semi-circular cylinder when two tandem semi-circular cylinders are arranged with "Back to Back" orientation (Figure 4(d)) at gap ratios of 3 and 5. The peak amplitude of WIV at gap ratio of 5 is $61 \%$ larger than that of WIV at gap ratio of 3 . However, when the cylinders are set as Figure 4 (a) (c) and (d), the downstream one almost stays static. For two tandem square cylinders (Figures 6 (e) and (f)), the vibration response of the downstream cylinder is lower than 0.1 at both gap ratios. Thus, the present results show that triangular and square cylinders arranged in tandem may not be the proper options for energy harnessing. In addition, it can be seen that the downstream vibrating circular cylinder has the largest amplitude without upper limit in all cases, with amplitude of about 1 at gap ratio of 3 and 0.9 at gap ratio of 5 at the maximum reduced velocity. Therefore, circular cylinders arranged in tandem can be considered as the best option for energy harnessing based on WIV.

\section{Conclusions}

The vibration responses of four cylinders with different cross-sectional geometries are examined in a wind tunnel. The following conclusions can be drawn from the tests conducted:

1) For single cylinders, the amplitudes of triangular and square cylinders are much larger than that of the circular and semicircular cylinders. Moreover, the amplitude is getting higher with the increase of the wind speed. Thus, the square and the triangular cylinders are better options than the circular and semi-circular cylinders for energy harnessing based on VIV. For two cylinders arranged in tandem at gap ratios of 3 and 5, circular cylinders arranged in tandem may be the best option for energy harnessing when considering WIV.

2) The vibration amplitude of two semi-circular cylinders which are set "Back to Back" at gap ratio 5 has no upper limit; it means that the lock-on region has been extended. However, single semi-circular has its own synchronization region and limited vibration amplitude. Thus, the semi-circular cylinders arranged in tandem should be paid more attention for the future study.

3) No matter what the cross-section is, the WIV response of tandem cylinders is different from the VIV response of a single cylinder. What is more, the WIV response is related to the gap ratio, geometries of cylinder and setting orientation. 


\section{References}

1. Paidoussis M P, Price S J and de Langre E 2010 Fluid-structure interactions: cross-flow-induced instabilities (Cambridge University Press)

2. Bokaian A, Geoola F 1984 Wake-induced galloping of two interfering circular cylinders Journal of Fluid Mechanics 146 383-415

3. Bernitsas M M, Raghavan, $K$ Ben-Simon $Y$ and Garcia E M H 2006 VIVACE (Vortex induced vibration aquatic clean energy): a new concept in generation of clean and renewable energy from fluid flow Proc. of the 25th Int. Conf. on Offshore Mechanics and Arctic Engineering 2 pp 619637

4. Sun H, Ma C, Kim ES, Nowakowski G, Mauer E and Bernitsas M M 2017 Hydrokinetic energyconversion by two rough tandem-cylinders in flow induced motions: Effect of spacing andstiffness Renewable Energy 107 61-80.

5. Khalak A and Williamson C H K 1996 Dynamics of a hydroelastic cylinder with very low mass and damping J. Fluid Struct. 10 455-72

6. Feng C 1968 The measurement of vortex induced effects in flow past stationary and oscillating circular and d-section cylinders $P h D$ thesis (University of British Columbia, Department of mechanical engineering)

7. Zdravkovich M 1987 The effects of interference between circular cylinders in cross flow Journal of fluids and structures 1 239-261 